\title{
The Prevalence of Chronic Physical Diseases Comorbid with Depression among Different Sex and Age Groups in South Korea: A Population-Based Study, 2007-2014
}

\author{
Se Jin Park', Seungyeon Hong ${ }^{1}$, Hyesue Jang ${ }^{1}$, Jung Won Jang ${ }^{1,2}$, \\ Boram Yuk ${ }^{1}$, Chul Eung Kim ${ }^{1}$, and Subin Park ${ }^{1 凶}$ \\ ${ }^{1}$ Department of Research Planning, Mental Health Research Institute, National Center for Mental Health, Seoul, Republic of Korea \\ ${ }^{2}$ Department of Health Sciences, Graduate School, Hanyang University, Seoul, Republic of Korea
}

\begin{abstract}
Objective People with depression often suffer from comorbid, chronic physical diseases. Little is known about how demographic characteristics such as age and sex influence the prevalence of chronic physical diseases comorbid with depression. Therefore, this study aimed to explore the age and sex differences in the prevalence of diverse, chronic physical diseases comorbid with depression.

Methods This cross-sectional survey were conducted with the participants $(n=45,598)$ of the Korean National Health and Nutrition Examination Survey (KNHANES). Using log-binomial regression, age adjusted prevalence ratios (APR) of chronic physical diseases of participants with depression and those without depression were estimated for each sex and age group.

Results The APR of most chronic physical diseases were significantly higher among respondents with depression than those without depression. Chronic physical diseases that showed the highest APR were asthma in adult male respondents $(A P R=3.46)$ and adult female respondents $(\mathrm{APR}=2.19)$ and chronic renal failure in elderly male respondents $(\mathrm{APR}=8.36)$ and elderly female respondents (APR=1.94).

Conclusion Prevalence ratios of the chronic physical diseases comorbid with depression differed according to sex and age groups. Collaborative care strategies should be designed according to demographic characteristics of the population.
\end{abstract}

Psychiatry Investig 2018;15(4):370-375

Key Words Depression, Chronic physical disease, Comorbidity, Prevalence.

\section{INTRODUCTION}

Depression is a mood disorder frequently causing impairment in many areas of functioning, and has a relatively high prevalence ranging from $2 \%$ to $15 \% .{ }^{1}$ The medical and economic burden of depression is considerable because major depression is a leading cause of morbidity, mortality, and disability. ${ }^{2}$ People with depression often suffer from comorbid chronic physical conditions such as hypertension, ${ }^{3}$ stroke, ${ }^{4}$ asthma, ${ }^{5}$ and arthritis. ${ }^{6}$ Depression is known to increase the

Received: February 28, 2017 Revised: August 16, 2017

Accepted: September 11, 2017

$\square$ Correspondence: Subin Park, MD, PhD

Department of Research Planning, Mental Health Research Institute, National Center for Mental Health, 127 Yongmasan-ro, Gwangin-gu, Seoul 04933, Republic of Korea

Tel: +82-2-2204-0108, Fax: +82-2-2204-0393, E-mail: subin-21@hanmail.net

(c) This is an Open Access article distributed under the terms of the Creative Commons Attribution Non-Commercial License (http://creativecommons.org/licenses/by$\mathrm{nc} / 4.0$ ) which permits unrestricted non-commercial use, distribution, and reproduction in any medium, provided the original work is properly cited. risk for chronic diseases, and may worsen the course of these diseases. ${ }^{7.8}$ Possible reasons for such high comorbidity among the patients with depression include unhealthy lifestyle related to the depressive state such as smoking, poor adherence to medical regimens, and biological impact of depression on proinflammatory factors, the hypothalamic-pituitary axis, autonomic nervous system, and metabolic factors. ${ }^{79,10}$

The relationship between depression and chronic physical diseases can further be associated with other factors such as age and sex. Previous studies have reported that the impact of depression on health conditions such as chronic medical disorders and health-related quality of life is unequally distributed among different population groups of age, sex, marital status, economy, and employment. ${ }^{2,11}$ Despite these differences in the association between depression and health outcomes across the demographic groups, little is known about the prevalence of chronic physical diseases comorbid with depression among the different demographic groups. Therefore, this study 
aimed to estimate the prevalence of diverse, comorbid, chronic physical diseases across sex and age groups using a Korean representative nationwide sample.

\section{METHODS}

\section{Study sample and measures}

This study used the Korean National Health and Nutrition Examination Survey (KNHANES). The KNHANES included cross-sectional health interview survey, health examination survey, and nutrition survey conducted with a representative sample of non-institutionalized Korean civilian population. ${ }^{12}$ The Institutional Review Board of Korea Centers for Disease Control and Prevention approved the protocol (2007-02CON04-P, 2008-04EXP-01-C, 2009-01CON-03-2C, 2010-02CON21-C, 2011-02CON-06-C, 2012-01EXP-01-2C, 2013-07CON03-4C, and 2013-12EXP-03-5C). We included the data of 45,598 respondents who participated in both the health interviews and examinations of a total of 50,094 respondents aged 19 years or older, between 2007 and 2014.

Data about depression, arthritis, angina or myocardial infarction, stroke, tuberculosis, asthma, chronic renal failure, atopic dermatitis, thyroid gland disease, cancer (stomach, liver, colorectal, and breast) and cirrhosis of the liver were collected using questions about being diagnosed; for example, "Have you ever been diagnosed with depression by a physician?". ${ }^{2}$

Metabolic syndrome was measured in the health examination. These health examinations were conducted in local community health centers and clinics, and standardized protocols were used to assess anthropometry, blood pressure, and laboratory indices. Metabolic syndrome was considered to be present if at least 3 of the following 5 criteria were satisfied: 1) abdominal obesity (waist circumference of $\geq 85 \mathrm{~cm}$ ), measured using a Korean-specific standard; 2) high blood pressure (SBP of $\geq 130 \mathrm{~mm} \mathrm{Hg}$ or DBP of $\geq 85 \mathrm{~mm} \mathrm{Hg}$ ); 3) a high TG level ( $\geq 150 \mathrm{mg} / \mathrm{dL}$ ); 4 ) a low HDL-C level ( $<50 \mathrm{mg} / \mathrm{dL}$ ); and 5) elevated FPG $(\geq 100 \mathrm{mg} / \mathrm{dL})$. We used the version of the National Cholesterol Education Program's Adult Treatment Panel III criteria recommended by the American Heart Association and the National Heart, Lung, and Blood Institute. ${ }^{12}$

We defined adults as respondents aged 19-64 years, and elderly as respondents aged 65 years and above.

\section{Statistical analysis}

The age-standardized prevalence rates for comorbidity of depression with chronic physical diseases were estimated using the standard population from the Population Projections for Korea: 2010-2016 (based on the 2010 census). Log-binomial regression was performed to estimate age adjusted prevalence ratios (APRs) of chronic physical diseases for respondents with depression and those without depression. We used SAS software version 9.4 (SAS institute Inc., Cary, NC, USA) for all analyses.

\section{RESULTS}

Of the total population $(n=45,598), 3.7 \%(n=1,860)$ had been diagnosed with depression by physicians. Of the adults aged $19-64$ years $(n=34,837), 3.4 \%(n=1,289)$ and $5.1 \%(n=$ $571)$ of the elderly aged 65 years and above $(n=10,761)$ were diagnosed with depression. Of male respondents $(n=19,263)$, $1.8 \%(\mathrm{n}=355)$ and $5.4 \%(\mathrm{n}=1,505)$ of female respondents $(\mathrm{n}=$ 26,335) reported that they had depression (Table 1).

As shown in Tables 2 and 3, age-standardized prevalence rates for metabolic syndrome were high among respondents with depression in all sex and age-groups. In addition to the aforementioned diseases, asthma in adult male respondents (7.53\%, $\mathrm{SE}=2.13 \%)$, thyroid gland disease in adult female respondents $(6.92 \%, \mathrm{SE}=0.89 \%)$, tuberculosis in elderly male respondents (5.35\%, $\mathrm{SE}=1.52 \%)$, and asthma in elderly female respondents ( $4.19 \%, \mathrm{SE}=0.78 \%$ ) showed high prevalence rates among respondents with depression.

The APR of most chronic physical diseases were significantly higher among respondents with depression than those without depression. We specifically focused on the chronic physical diseases that were highly associated with depression (with APR higher than 2). Arthritis showed the highest APR of 2.41 $(95 \% \mathrm{CI}=2.27-2.40)$ in the adult male group, followed by the elderly male group (APR=2.06, 95\% CI=2.03-2.09). The APR of arthritis was higher in adult respondents than elderly respondents and in men than women. Similarly, asthma showed the highest APR of 3.46 (95\% CI=3.42-3.51) in the adult male group, followed by the elderly male group (APR $=2.33,95 \%$ $\mathrm{CI}=2.27-2.40$ ). Like arthritis, APR of this disease was higher in adult respondents than elderly respondents and in men than women. The APR of chronic renal failure was remarkably higher in elderly male group than other groups. There was a difference in the APR of chronic renal failure between the elderly group and adult group among men $(\mathrm{APR}=8.36,95 \% \mathrm{CI}=$ 8.01-8.72 vs. $\mathrm{APR}=1.57,95 \% \mathrm{CI}=1.49-1.66)$, but not among women $(\mathrm{APR}=1.94,95 \% \mathrm{CI}=1.87-2.02$ vs. $\mathrm{APR}=2.05,95 \%$ $\mathrm{CI}=1.99-2.10)$. Thyroid gland disease also showed higher APR in elderly male group than other groups. There was a difference in the APR of thyroid gland disease between the elderly group and adult group among men $(\mathrm{APR}=3.60,95 \% \mathrm{CI}=$ 3.44-3.77 vs. $\mathrm{APR}=1.45,95 \% \mathrm{CI}=1.40-1.50$ ), but not among women $(\mathrm{APR}=1.22$, 95\% $\mathrm{CI}=1.20-1.24$ vs. $\mathrm{APR}=1.44,95 \%$ $\mathrm{CI}=1.43-1.46)$. Cirrhosis of the liver also showed higher APR in the elderly male group than other groups. There was a difference in the APR of cirrhosis of the liver between the elder- 
Table 1. Demographic characteristics of the samples according to presence of lifetime depression diagnosis among Koreans (aged 19 years or over)

\begin{tabular}{|c|c|c|c|c|}
\hline & Total & Depression $(\mathrm{N}=1,860)$ & Non-depression $(\mathrm{N}=14,504)$ & p-value* \\
\hline Age groups, year, N (\%) ${ }^{\dagger}$ & & & & $<0.0001$ \\
\hline $19-64$ & $34,837(85.8)$ & $1,289(3.4)$ & $33,548(96.6)$ & \\
\hline$\geq 65$ & $10,761(14.2)$ & $571(5.1)$ & $10,190(94.9)$ & \\
\hline Sex, $N(\%)^{\dagger}$ & & & & $<0.0001$ \\
\hline Male & $19,263(49.2)$ & $355(1.8)$ & $18,908(98.2)$ & \\
\hline Female & $26,335(50.8)$ & $1,505(5.4)$ & $24,830(94.6)$ & \\
\hline Education, $\mathrm{N}(\%)^{\dagger}$ & & & & $<0.0001$ \\
\hline$\geq$ High school & $28,364(71.2)$ & $832(2.8)$ & $27,532(97.2)$ & \\
\hline$<$ High school & $17,088(28.8)$ & $1,019(5.8)$ & $16,069(94.2)$ & \\
\hline Arthritis & $6,180(9.7)$ & $535(22.8)$ & $5,645(9.2)$ & $<0.0001$ \\
\hline Angina or myocardial infarction & $1,132(1.8)$ & $87(3.1)$ & $1,045(1.7)$ & $<0.0001$ \\
\hline Stroke & $1,001(1.5)$ & $76(2.7)$ & $925(1.5)$ & $<0.0001$ \\
\hline Tuberculosis & $2,255(4.4)$ & $116(5.4)$ & $2,139(4.4)$ & $<0.0001$ \\
\hline Asthma & $1,480(2.9)$ & $133(7.0)$ & $1,347(2.8)$ & $<0.0001$ \\
\hline Chronic renal failure & $196(0.4)$ & $22(0.9)$ & $174(0.3)$ & $<0.0001$ \\
\hline Atopic dermatitis & $1,112(2.9)$ & $55(2.9)$ & $1,057(2.9)$ & 0.9983 \\
\hline Thyroid gland disease & $1,655(3.0)$ & $140(6.2)$ & $1,515(2.8)$ & $<0.0001$ \\
\hline Cancer $^{\ddagger}$ & $740(1.2)$ & $47(2.3)$ & $693(1.2)$ & 0.0015 \\
\hline Cirrhosis of the liver & $120(0.2)$ & $8(0.3)$ & $112(0.2)$ & 0.4688 \\
\hline Metabolic syndrome & $13,965(28.5)$ & $615(30.9)$ & $13,350(28.4)$ & 0.0644 \\
\hline
\end{tabular}

*statistical significance of difference between groups with and without depression, $\%$ \% population weighted proportions, fany cancer includ- $^{\circ}$ ing stomach, liver, colorectal, and breast cancers

ly group and adult group among both men (APR $=2.27,95 \%$ $\mathrm{CI}=2.13-2.42$ vs. $\mathrm{APR}=1.69,95 \% \mathrm{CI}=1.60-1.78)$ and women (APR $=1.36,95 \% \mathrm{CI}=1.28-1.45$ vs. $\mathrm{APR}=0.53,95 \% \mathrm{CI}=0.49$ $0.58)$ Stroke (APR $=1.79,95 \% \mathrm{CI}=1.75-1.82)$ and cancer (APR=1.66, 95\% CI=1.64-1.69) had higher APRs in the adult female group than other groups (Table 2 and 3 ).

Among the respondents with depression, though prevalence rates of chronic renal failure were lower than other physical diseases, APRs of this disease were fairly high, compared to those without depression, showing a maximum of 8 -fold increase in elderly male respondents with depression compared to those without depression. On the contrary, the prevalence rates of metabolic syndrome were very high, but APRs of this disease was relatively low in respondents with depression because of its high prevalence among both, respondents with and those without depression (Table 2 and 3 ).

\section{DISCUSSION}

To our knowledge, this study is the first to present APRs of diverse, chronic physical diseases related to depression among sex and age groups separately. Our results have shown that the prevalence of most chronic physical diseases are significantly higher among respondents with depression than those without depression and associations between depression and chronic physical diseases is unequally distributed across sex and agegroups.

The noticeable result was that elderly male respondents with depression were much more vulnerable to chronic physical diseases (i.e., arthritis, angina, asthma, renal failure, thyroid gland disease, and cirrhosis of the liver) than elderly female respondents with depression, although the prevalence of depression in elderly female respondents was about three times higher than in elderly male respondents $(6.9 \%$ and $2.4 \%$ respectively). There were more chronic physical diseases that showed an APR higher than 2 in elderly male respondents than any other groups. Such diseases include arthritis, asth$\mathrm{ma}$, chronic renal failure, thyroid gland disease, and cirrhosis (APRs of 2.06, 2.33, 8.36, 3.60, and 2.27, respectively). Our finding is consistent with the results of a Korean study which found that the association between depressive symptoms and a number of physical illnesses is stronger in older male respondents than older female respondents and having one or more physical illnesses increased the risk of suicidal ideation in older male respondents, but not in older female respondents. ${ }^{13}$

Such a high comorbidity of depression with physical diseas- 

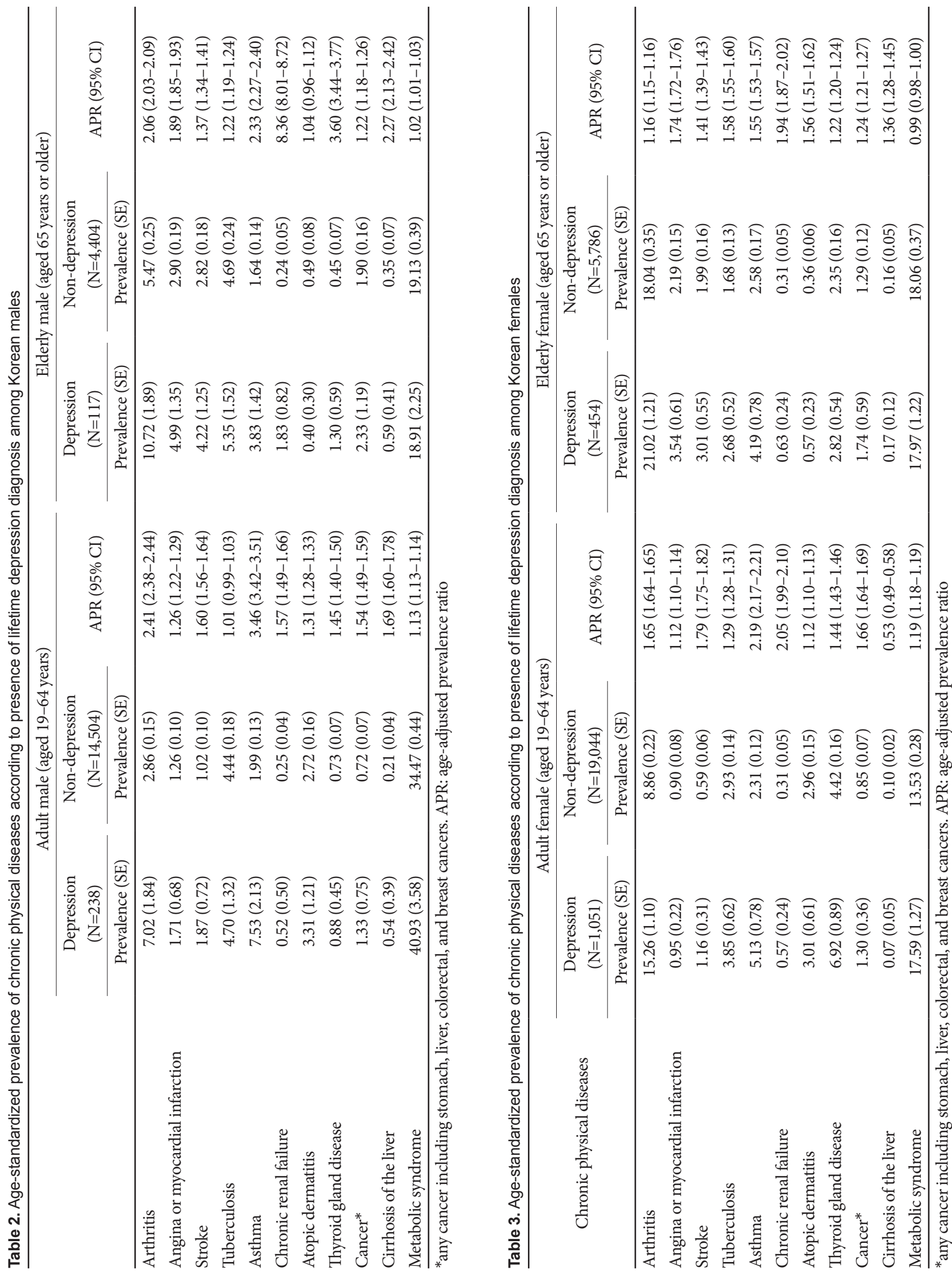
es among older male respondents may be partially explained by the vulnerability of older men in a post-retirement environment and widowhood. Isolation and loneliness related to changes in role identity after retirement or loss of a partner or a relationship was found to be more detrimental for elderly male subjects than elderly female subjects. ${ }^{14}$ Alpass and Neville ${ }^{15}$ suggested that loneliness in older men negatively affects relationships and independent functioning and may eventually lead to depression. Such detrimental effects of loneliness and impoverished relationships in older men could affect their regular functioning in terms of eating a healthy diet and taking medication, which can also adversely affect their physical health. ${ }^{16}$ On the contrary, disability due to physical illness may increase the risk of loneliness or depression. ${ }^{7,17}$ The links between loneliness, depression, and physical illness are complex, so the causal mechanisms have been difficult to demonstrate. $^{18}$

Especially, chronic renal failure has shown the highest APR ( $\mathrm{APR}=8.36,95 \% \mathrm{CI}=8.01-8.72)$ in elderly male respondents with depression among all sex and age groups in our findings. Little is known about why chronic renal failure is disproportionately common in elderly men with depression compared to those without depression. However, the facts that the annual incidence of chronic renal failure increases rapidly with advancing age, and is three times higher in male patients than female patients who are 75 years old or more ${ }^{19}$ and that deterioration of renal function in patients with chronic renal disease is more rapid in male patients than in female patients ${ }^{20}$ might be related to the high comorbidity of chronic renal failure in elderly men with depression. Most studies reported the development of depression among patients with chronic renal diseases. ${ }^{21}$ However, a recent review showed the bidirectional and multifactorial relationship between kidney disease and depression. ${ }^{22}$ Bautovich et al. have suggested that depression can increase the risk of chronic kidney disease as it has biological consequences, such as increased inflammation, and negative self-care behaviors, such as sedentary lifestyles and poor compliance with medical care. Furthermore, such comorbidity of depression with chronic renal disease is highly associated with an increased risk of adverse outcomes such as hospitalization, death, suicide ${ }^{23-25}$ and hence careful treatment and follow-up are required.

Previous studies have reported the comorbidity between depression and arthritis, especially rheumatoid arthritis. In a cross-sectional study, almost three-fourths (71.5\%) of rheumatoid arthritis patients were found to have depression, and the level of depression was strongly associated with rheumatoid arthritis disease activity. ${ }^{6}$ A longitudinal study in Taiwan investigated the strong bidirectional association between depression and rheumatoid arthritis, and found hazard ratios of
1.69 (95\% CI=1.51-1.87) of depression in rheumatoid arthritis and hazard ratios of 1.65 (95\% CI=1.41-1.77) of rheumatoid arthritis in depression. ${ }^{26}$ This study reported that neurotransmitter deregulation and dysfunctional intracellular signaling are possible mechanisms linking psychological stress with immune-related diseases. ${ }^{26}$ In the present study, interestingly, although the prevalence rate of depression comorbid with arthritis was about two times higher in female respondents than male respondents in both adult and elderly groups, the APR of arthritis was higher in male respondents with depression than female respondents with depression. Additionally, adult respondents with depression had higher APR of arthritis than elderly respondents with depression. Consistent with our findings, a review of comorbidities with rheumatoid arthritis reported that there are sex-specific differences in the prevalence of comorbidities of arthritis and depression, and thus, suggested that sex-specific aspects should be taken in to consideration in the treatment of these comorbidities. ${ }^{27}$ In our study, respondents with depression in all sex and age groups were twice as likely to have asthma than those without depression with the exception of the elderly female group, with the highest APR of asthma occurring among adult male respondents with depression. The APR of asthma was higher in respondents with depression among adult groups than elderly groups. Consistent with our findings, a cohort study reported that incidence of asthma was 1.91 times higher in patients with depression than in those without depression, and younger patients aged 20-49 years had a higher hazard ratio of asthma than the older patients aged 65 years or more. ${ }^{28} \mathrm{~A}$ recent meta-analysis that involved eight cohorts reported that depression was associated with a $43 \%$ increased risk of developing asthma. ${ }^{29}$ It is reported that depressing stimuli may cause airway instability and can lead to asthma. ${ }^{30}$ Van Lieshout et al. ${ }^{5}$ further suggested that malfunctioning of the hypothalamic pituitary adrenal axis may predispose people to both depression and asthma and bring about similar changes in the immune, autonomic nervous, and other important systems which may also contribute to the comorbidity of depression with asthma.

There were several limitations that should be considered when interpreting the results. First, the cross-sectional design of this study did not allow the investigation of the temporal or causal relationship between depression and a chronic physical disease. The association between depression and chronic physical diseases is increasingly considered to be bidirectional. ${ }^{7}$ Second, the diagnosis of depression was made based on self-reported diagnosis using a single question, rather than a structured diagnostic interview or a standardized assessment tool; therefore, there may be some cases of misclassification of depression, although self-reported diagnosis is reported to 
be largely congruent with clinical record data. ${ }^{31}$ Future studies using standardized assessment or medical examination are needed to confirm our findings.

Despite these limitations, the strength of our study was that we used a large, nationally representative sample, and showed the differences in the prevalence of diverse, chronic physical diseases comorbid with depression across sex and age groups, which, to our knowledge, had not been established through previous studies. Our findings suggested that the prevalence of chronic physical diseases that are more likely to occur in patients with depression was different in different groups in the population. For example, older male respondents with depression showed a much higher risk of having chronic renal failure than the other groups. Thus, professionals in the fields of mental and physical health should realize the seriousness of physical diseases comorbid with depression, and collaborative care strategies should be designed according to demographic characteristics of the population. Future studies will be needed to further explore the depth of depression's effect on a chronic disease according to the characteristics of the population.

\section{Acknowledgments}

This study was supported by an Intramural Research Grant (No R2017A) from the National Center for Mental Health, Ministry of Health \& Welfare, Republic of Korea.

\section{REFERENCES}

1. Moussavi S, Chatterji S, Verdes E, Tandon A, Patel V, Ustun B. Depression, chronic diseases, and decrements in health: results from the World Health Surveys. Lancet 2007;370:851-858.

2. Egede LE. Major depression in individuals with chronic medical disorders: prevalence, correlates and association with health resource utilization, lost productivity and functional disability. Gen Hosp Psychiatry 2007;29:409-416.

3. Abas M, Hotopf M, Prince M. Depression and mortality in a high-risk population. Br J Psychiatry 2002;181:123-128.

4. Morris PL, Robinson RG, Raphael B. Prevalence and course of depressive disorders in hospitalized stroke patients. Int J Psychiatry Med 1990;20:349-364.

5. Van Lieshout RJ, Bienenstock J, MacQueen GM. A review of candidate pathways underlying the association between asthma and major depressive disorder. Psychosom Med 2009;71:187-195.

6. Imran MY, Saira Khan EA, Ahmad NM, Farman Raja S, Saeed MA, Ijaz Haider I. Depression in Rheumatoid Arthritis and its relation to disease activity. Pak J Med Sci 2015;31:393-397.

7. Katon WJ. Epidemiology and treatment of depression in patients with chronic medical illness. Dialogues Clin Neurosci 2011;13:7-23.

8. Moussavi S, Chatterji S, Verdes E, Tandon A, Patel V, Ustun B. Depression, chronic diseases, and decrements in health: results from the World Health Surveys. Lancet 2007;370:851-858.

9. Gehi A, Haas D, Pipkin S, Whooley MA. Depression and medication adherence in outpatients with coronary heart disease: findings from the Heart and Soul Study. Arch Intern Med 2005;165:2508-2513.

10. Kim JH, Chang SM, Bae JN, Cho SJ, Lee JY, Kim BS, et al. Mentalphysical comorbidity in Korean adults: results from a nationwide gen- eral population survey in Korea. Psychiatry Investig 2016;13:496-503.

11. Isacson D, Bingefors K, von Knorring L. The impact of depression is unevenly distributed in the population. Eur Psychiatry 2005;20:205212.

12. Park SJ, Roh S, Hwang J, Kim HA, Kim S, Lee TK, et al. Association between depression and metabolic syndrome in Korean women: results from the Korean national health and nutrition examination survey (2007-2013). J Affect Disord 2016;205:393-399.

13. Jeon GS, Jang SN, Rhee SJ, Kawachi I, Cho SI. Gender differences in correlates of mental health among elderly Koreans. J Gerontol B Psychol Sci Soc Sci 2007;62:S323-S329.

14. Antonucci TC, Akiyama H. An examination of sex differences in social support among older men and women. Sex Roles 1987;17:737-749.

15. Alpass FM, Neville S. Loneliness, health and depression in older males. Aging Ment Health 2003;7:212-216.

16. Martikainen P, Valkonen T. Mortality after the death of a spouse: rates and causes of death in a large Finnish cohort. Am J Public Health 1996; 86:1087-1093.

17. Paul C, Ayis S, Ebrahim S. Psychological distress, loneliness and disability in old age. Psychol Health Med 2006;11:221-232.

18. Courtin E, Knapp M. Social isolation, loneliness and health in old age: a scoping review. Health Soc Care Community 2017;25:799-812.

19. Jungers P, Chauveau P, Descamps-Latscha B, Labrunie M, Giraud E, Man NK, et al. Age and gender-related incidence of chronic renal failure in a French urban area: a prospective epidemiologic study. Nephrol Dial Transplant 1996;11:1542-1546.

20. Silbiger SR, Neugarten J. The impact of gender on the progression of chronic renal disease. Am J Kidney Dis 1995;25:515-533.

21. Hedayati SS, Finkelstein FO. Epidemiology, diagnosis, and management of depression in patients with CKD. Am J Kidney Dis 2009;54: 741-752.

22. Bautovich A, Katz I, Smith M, Loo CK, Harvey SB. Depression and chronic kidney disease: a review for clinicians. Aust N Z J Psychiatry 2014;48:530-541.

23. Chen CK, Tsai YC, Hsu HJ, Wu IW, Sun CY, Cho CC, et al. Depression and suicide risk in hemodialysis patients with chronic renal failure. Psychosomatics 2010;51:528.e6.

24. Hedayati SS, Minhajuddin AT, Afshar M, Toto RD, Trivedi MH, Rush AJ. Association between major depressive episodes in patients with chronic kidney disease and initiation of dialysis, hospitalization, or death. JAMA 2010;303:1946-1953.

25. Lopes AA, Bragg J, Young E, Goodkin D, Mapes D, Combe C, et al. Depression as a predictor of mortality and hospitalization among hemodialysis patients in the United States and Europe. Kidney Int 2002; 62:199-207.

26. Lu MC, Guo HR, Lin MC, Livneh H, Lai NS, Tsai TY. Bidirectional associations between rheumatoid arthritis and depression: a nationwide longitudinal study. Sci Rep 2016;6:20647.

27. Albrecht K. [Gender-specific differences in comorbidities of rheumatoid arthritis]. Z Rheumatol 2014;73:607-614.

28. Shen T, Lin C, Liao C, Wei C, Sung F, Kao C. Major depressive disorder is associated with subsequent adult-onset asthma: a population-based cohort study. Epidemiol Psychiatr Sci 2017;26:664-671.

29. Gao YH, Zhao HS, Zhang FR, Gao Y, Shen P, Chen RC, et al. The relationship between depression and asthma: a meta-analysis of prospective studies. PLoS One 2015;10:e0132424.

30. Lehrer P, Feldman J, Giardino N, Song HS, Schmaling K. Psychological aspects of asthma. J Consult Clin Psychol 2002;70:691-711.

31. Kehoe R, Wu SY, Leske MC, Chylack LT Jr. Comparing self-reported and physician-reported medical history. Am J Epidemiol 1994;139: 813-818. 